\title{
Correlating nonresonant di-electron searches at the LHC to the Cabibbo-angle anomaly and lepton flavor universality violation
}

\author{
Andreas Crivellin $\oplus^{*}$ \\ CERN Theory Division, CH-1211 Geneva 23, Switzerland, \\ Physik-Institut, Universität Zürich, Winterthurerstrasse 190, CH-8057 Zürich, Switzerland, \\ and Paul Scherrer Institut, CH-5232 Villigen PSI, Switzerland \\ Claudio Andrea Manzari $\oplus^{\dagger}$ and Marc Montull ${ }^{\ddagger}$ \\ Physik-Institut, Universität Zürich, Winterthurerstrasse 190, CH-8057 Zürich, Switzerland \\ and Paul Scherrer Institut, $\mathrm{CH}-5232$ Villigen PSI, Switzerland
}

(Received 24 March 2021; accepted 8 November 2021; published 14 December 2021)

\begin{abstract}
In addition to the existing strong indications for lepton flavor universality violation in low-energy precision experiments, the CMS Collaboration at CERN recently released an analysis of nonresonant dilepton pairs which could constitute the first sign of lepton flavor universality violation in high-energy searches at the LHC. In this article, we show that the Cabibbo-angle anomaly, an (apparent) violation of first row and column Cabibbo-Kobayashi-Maskawa $(\mathrm{CKM})$ matrix unitarity with $\approx 3 \sigma$ significance, and the CMS result can be correlated and commonly explained in a model-independent way by the operator $\left[Q_{\ell q}^{(3)}\right]_{1111}=\left(\bar{\ell}_{1} \gamma^{\mu} \sigma^{I} \ell_{1}\right)\left(\bar{q}_{1} \gamma_{\mu} \sigma^{I} q_{1}\right)$. This is possible without violating the bounds from the nonresonant dilepton search of ATLAS (which interestingly also observed slightly more events than expected in the electron channel) nor from $R(\pi)=\pi \rightarrow \mu \nu / \pi \rightarrow e \nu$. We find a combined preference for the new physics hypothesis of $4.5 \sigma$ and predict $1.0004<R(\pi)<1.0009$ (95\% C.L.) which can be tested in the near future with the forthcoming results of the PEN experiment.
\end{abstract}

DOI: $10.1103 /$ PhysRevD.104.115016

\section{INTRODUCTION}

The Standard Model (SM) of particle physics has been very successfully tested with great precision in the last decades. Nonetheless, it is clear that the SM cannot be the ultimate fundamental theory of physics. For example, it has to be extended to account for dark matter and neutrino masses, but neither the scale nor the concrete nature of the additional particles necessary to explain these observations is unambiguously established. Fortunately, in the flavor sector intriguing (indirect) hints for physics beyond the SM at the (multi) $\mathrm{TeV}$ scale have been collected in the last years. In particular, lepton flavor universality has been tested extensively, unveiling intriguing signs of beyond the SM physics in $b \rightarrow s \ell^{+} \ell^{-}$[1-8] data, $b \rightarrow c \tau \nu$ [9-14] transitions, and the anomalous magnetic moment of the

\footnotetext{
*andreas.crivellin@cern.ch

†claudioandrea.manzari@physik.uzh.ch

"marc.montull@psi.ch
}

Published by the American Physical Society under the terms of the Creative Commons Attribution 4.0 International license. Further distribution of this work must maintain attribution to the author(s) and the published article's title, journal citation, and DOI. Funded by SCOAP ${ }^{3}$. muon [15] with a significance of $>5 \sigma$ [16-27], $>3 \sigma$ [28-32], and 3.7 $\sigma$ [33], respectively. Furthermore, the Cabibbo-angle anomaly, a deficit in first row (and first column) CKM unitarity with a significance of at least $3 \sigma$ [34-37], can also be explained within the framework of lepton flavor universality violation (LFUV) beyond the SM $[37,38]$. However, until recently, no hints of LFUV at highenergy searches at the LHC had emerged.

This changed when CMS recently reported the results of a first test of LFUV in nonresonant dilepton searches by measuring the dimuon to dielectron ratio [39], observing an excess in the electron channel. Interestingly, ATLAS also found slightly more electron events than expected in the signal region in the search for quark-lepton contact interactions [40] and HERA reported more electron events than expected as well [41], even though the bounds are not competitive with the ones from the LHC. Even though CMS actually states that no significant tension with the SM prediction is observed, the surplus of events appears in the bins with high invariant mass of the electron pair, as expected in case of heavy new physics that can be parametrized in terms of effective 2-quark-2-lepton operators. Therefore, an investigation of the implications of this excess is very interesting, and in this article we want to show that the Cabibbo-angle anomaly (CAA) can be model-independently 
correlated to nonresonant dielectron searches. In fact, assuming that the Cabbibo-angle anomaly is explained by a direct new physics contribution to beta decays, a signal in dielectron production is even predicted, whose size turns out to agree with the data reported by CMS and is compatible with the ATLAS bounds. In addition, such an explanation predicts an observable effect in $R(\pi)=\pi \rightarrow$ $\mu \nu / \pi \rightarrow e \nu$ (defined at the amplitude level) which perfectly agrees with the current data and can be soon tested by the forthcoming results from the PEN [42] and PiENu [43] experiments.

In the next section we define our setup and discuss the different observables. We combine these observables and show the results of the global analysis in Sec. III before we conclude and give an outlook in Sec. IV.

\section{SETUP AND OBSERVABLES}

As outlined in the Introduction, the Cabbibo-angle anomaly is the deficit found in first row and first column CKM matrix unitarity [34-37]. The tension significantly depends on the radiative corrections to superallowed $\beta$ decays [36,44-50] and on the treatment of the $K_{\ell 2}$ and $K_{\ell 3}$ decays [51] as well as the constraints from $\tau$ decays [52] (see Ref. [38] for more details). However, a significance of around $3 \sigma$ should give a realistic estimate of the current situation. For definiteness we use the result of Ref. [53]

$$
\begin{aligned}
& \left|V_{u d}\right|^{2}+\left|V_{u s}\right|^{2}+\left|V_{u b}\right|^{2}=0.9985(5), \\
& \left|V_{u d}\right|^{2}+\left|V_{c d}\right|^{2}+\left|V_{t d}\right|^{2}=0.9970(18) .
\end{aligned}
$$

Note that even though the deficit in the first column CKM unitarity is less significant than the one of the first row, it suggests that, if the deficits were due to beyond Standard Model (BSM) effects they would likely be related to $\beta$ decays and therefore to $V_{u d}$. For the numerical analysis, we will only use the relation for the first row due to its higher precision.

There are several possibilities to account for the Cabbibo-angle anomaly [54]. For instance, via modified $W$-quark couplings [34,55], a modified $W-\mu \nu_{\mu}$ coupling [37,38,56-61], a tree-level contribution to the muon decay $[34,62,63]$, or by a tree-level effect in beta decays [64]. Since we aim at connecting the Cabbibo-angle anomaly to the CMS measurement we will focus on the latter possibility in which case only one operator, in the basis of Ref. [65],

$$
\left[Q_{\ell q}^{(3)}\right]_{1111}=\left(\bar{\ell}_{1} \gamma^{\mu} \sigma^{I} \ell_{1}\right)\left(\bar{q}_{1} \gamma_{\mu} \sigma^{I} q_{1}\right)
$$

(where $\sigma^{I}$ are the Pauli matrices) is capable of explaining the Cabbibo-angle anomaly without violating other bounds, in particular those set by $R(\pi)=\pi \rightarrow \mu \nu / \pi \rightarrow$ $e \nu$ [54]. This four-fermion operator generates effects in the neutral and charged-current processes after electroweak symmetry breaking via the Lagrangian

$$
\begin{aligned}
\mathcal{L}= & \mathcal{L}_{\mathrm{SM}}+\left[C_{\ell q}^{(3)}\right]_{1111}\left[\left(\bar{d} \gamma^{\mu} P_{L} d-\bar{u} \gamma^{\mu} P_{L} u\right) \bar{e} \gamma_{\mu} P_{L} e\right. \\
& +\left(\bar{u} \gamma^{\mu} P_{L} u-\bar{d} \gamma^{\mu} P_{L} d\right) \bar{\nu} \gamma_{\mu} P_{L} \nu \\
& \left.+2\left(\bar{d} \gamma^{\mu} P_{L} u \bar{\nu} \gamma_{\mu} P_{L} e+\bar{u} \gamma^{\mu} P_{L} d \bar{e} \gamma_{\mu} P_{L} \nu\right)\right],
\end{aligned}
$$

where we omitted CKM matrix elements. Note, that in principle, after CKM rotations, we could get effects in processes like $K \rightarrow \mu \nu / K \rightarrow e \nu$ or $K \rightarrow \pi \nu \nu$. However, these bounds can be avoided by assuming that $\left[C_{\ell q}^{(3)}\right]_{11 k l}$ is aligned to the down basis or flavor universality in the quark sector (i.e., $\left[C_{\ell q}^{(3)}\right]_{11 k l}=\delta_{k l}\left[C_{\ell q}^{(3)}\right]_{1111}$ ). A nonzero Wilson coefficient of the operator $\left[Q_{\ell q}^{(3)}\right]_{1111}$ modifies the CKM unitarity relations. Using Eq. (1) we find that the best fit point for the Wilson coefficient is

$$
\left[C_{\ell q}^{(3)}\right]_{1111}=1.22(4) /(10 \mathrm{TeV})^{2} .
$$

$\left.Q_{\ell q}^{(3)}\right]_{1111}$ also contributes to nonresonant dielectron production at the LHC, which is tailored to search for heavy new physics that is above the direct production reach $[66,67]$ and therefore can be parametrized in terms of an effective Lagrangian. The latest dilepton results from ATLAS and CMS are presented in Ref. [40] and Ref. [39], respectively.

Even though ATLAS does not claim any tension with the SM prediction, they observe $19 e^{+} e^{-}$events in the signal region for the case of constructive interference, as required by the CMS result, compared to an expectation of $12.4 \pm 1.9$ events. ${ }^{1}$ This leads to a limit that is weaker than expected but which agrees with the SM expectation within $2 \sigma$ once the statistical uncertainty is included. We have to recast the ATLAS limit on the Wilson coefficient since their bound is obtained for operators that have equal coupling to up and down quarks while $\left[Q_{\ell q}^{(3)}\right]_{1111}$ leads to a relative minus sign between them. ${ }^{2}$ Recasting the case with constructive interference with the SM we find that

$$
\left[C_{\ell q}^{(3)}\right]_{1111} \lesssim 1.4 /(10 \mathrm{TeV})^{2}
$$

at $95 \%$ C.L. We obtained this limit by using the differential parton-antiparton luminosities [69] which for partons $i, j=u, d, s, c, b$ are given by

\footnotetext{
${ }^{1}$ Here, the error includes only the systematic and not the statistical uncertainty. Note that in the latest analysis ATLAS also observed more electrons than expected [40], while in previous publications a slight deficit was found [68].

${ }^{2}$ Here we corrected Eq. (1) of Ref. [40] by a missing factor of 2. We thank the ATLAS Collaboration for confirming this typo.
} 


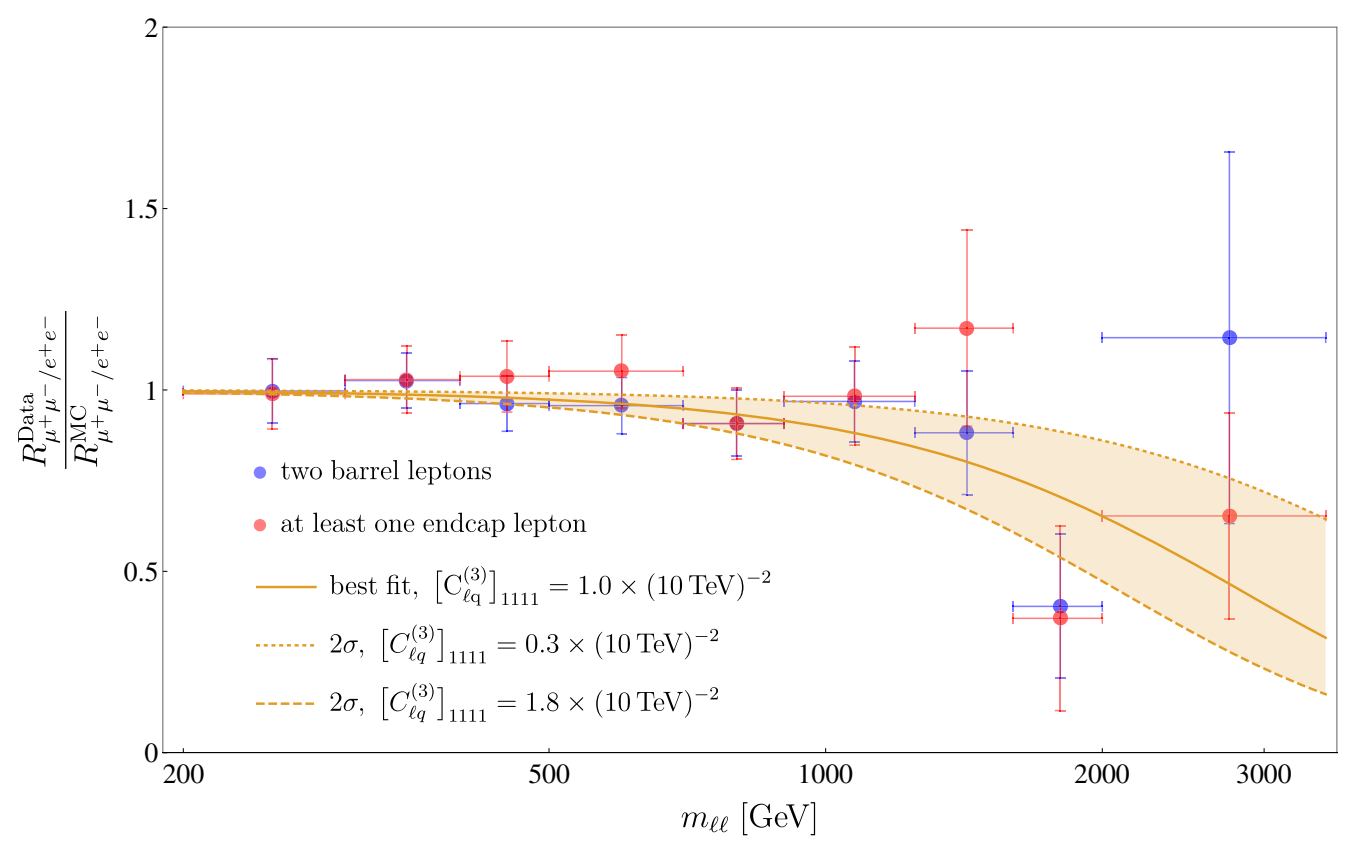

FIG. 1. Ratio of the differential dilepton production cross section in the dimuon and dielectron channels as a function of the lepton pair invariant mass for events with two barrel leptons (blue) and at least one lepton in the endcaps (red) [39]. The error bars include both statistical and systematic uncertainties. In dark orange is shown the predicted number of events for the best fit point of $\left[C_{\ell q}^{(3)}\right]_{1111}$ and the $2 \sigma$ region.

$$
\frac{d \mathcal{L}_{i \bar{j}}}{d \hat{s}}=\frac{1}{s} \int_{\tau}^{1} \frac{d x}{x} f_{i}(x, \sqrt{\hat{s}}) f_{j}\left(\frac{\tau}{x}, \sqrt{\hat{s}}\right)+(i \leftrightarrow \bar{j}),
$$

where $s(\hat{s})$ is the beam (parton) center of mass energy and $\tau=\hat{s} / s$. The integrated cross section over $\hat{s}$ can then be computed as

$$
\sigma=\sum_{i, \bar{j}} \int\left(\frac{d \hat{s}}{\hat{s}}\right)\left(\frac{d \mathcal{L}_{i \bar{j}}}{d \hat{s}}\right)\left(\hat{s} \hat{\sigma}_{i \bar{j}}\right),
$$

where $\hat{\sigma}_{a b}$ is the partonic cross section which is typically a function of $\hat{s}$. For the numerical evaluation we use the parton density function set NNPDF23LO, also employed e.g., by ATLAS analysis to generate the signal Drell-Yan process [40] with the help of the Mathematica package MANEPARSE [70]. We then computed at tree level the cross section for our new physics model, normalized to the SM one, as a function of the invariant mass of the lepton pair, integrated this over the invariant mass of the lepton pairs within the signal region $m_{\bar{\ell} \ell} \in[2.2,6] \mathrm{TeV}$ and compared it to the limits obtained from ATLAS.

CMS observed an excess in the dielectron cross section at high invariant lepton mass and computed the double ratio,

$$
R_{\mu^{+} \mu^{-} / e^{+} e^{-}}^{\mathrm{Data}} / R_{\mu^{+} \mu^{-} / e^{+} e^{-}}^{\mathrm{MC}},
$$

in order to reduce the uncertainties [71]. This means that they provide the relative signal strength for muons vs electrons, $R_{\mu^{+} \mu^{-}}^{\text {Data }} / e^{+} e^{-}$, divided to the SM expectation obtained from Monte Carlo simulations, $R_{\mu^{+} \mu^{-} / e^{+} e^{-}}^{\mathrm{MC}}$ Importantly, in this procedure the first bin is normalized to one in order to obtain the relative sensitivity to electrons and muons. Taking this into account, we find that the best fit value for the Wilson coefficient is

$$
\left[C_{\ell q}^{(3)}\right]_{1111} \approx 1.0 /(10 \mathrm{TeV})^{2},
$$

with $\quad \Delta \chi^{2} \equiv \chi^{2}-\chi_{\mathrm{SM}}^{2} \approx-10 \quad$ and $\quad 0.3 /(10 \mathrm{TeV})^{2} \lesssim$ $\left[C_{\ell q}^{(3)}\right]_{1111} \lesssim 1.8 /(10 \mathrm{TeV})^{2}$ at $95 \%$ C.L. as shown in Fig. 1. Here we followed the same approach outlined above for the ATLAS analysis and computed the ratio of the cross section in our model with respect to the tree-level $\mathrm{SM}$ one. Since the cross section is already dominated in the SM by left-handed amplitudes, we can assume that the changes in the angular distributions, affecting the CMS analysis, are small and can be safely neglected.

Finally we note that our operator also gives a lepton flavor universality violating effect which can be tested by charged pion decay. Defining the ratio $R(\pi)=\pi \rightarrow$ $\mu \nu / \pi \rightarrow e \nu$ at the amplitude level we have

$$
R(\pi) \simeq 1+0.0006\left[C_{\ell q}^{(3)}\right]_{1111} \times(10 \mathrm{TeV})^{2},
$$

which has to be compared with the experimental measurement [72-77] 


$$
R(\pi)=1.0010 \pm 0.0009
$$

Note that even though there is no deviation from the SM prediction, the $1 \sigma$ interval is in perfect agreement with the expectations from the CAA and CMS data.

\section{COMBINED ANALYSIS AND RESULTS}

Let us now perform the combined analysis of the observables discussed in the last section. In Fig. 2, we show the $\Delta \chi^{2}=\chi^{2}-\chi_{\text {SM }}^{2}$ for the CMS measurement of lepton flavor universality violation in nonresonant dilepton searches, the CAA and $R(\pi)$ separately, as well as the 95\% C.L. exclusion region from ATLAS. We compute the total $\Delta \chi^{2}$ function which has a minimum for $\left[C_{\ell q}^{(3)}\right]_{1111} \approx$ $1.1 /(10 \mathrm{TeV})^{2}$ of $\approx-20$, corresponding to a pull of $\approx 4.5 \sigma$ with respect to the SM. Note, that this minimum is well compatible with the $95 \%$ C.L. exclusion limit of ATLAS, which however cuts partially the $2 \sigma$ region preferred by $R(\pi)$, CMS, and the Cabbibo-angle anomaly. Treating the ATLAS exclusion as a hard cut, we therefore find that at 95\% C.L.

$$
0.6 /(10 \mathrm{TeV})^{2} \lesssim\left[C_{\ell q}^{(3)}\right]_{1111} \lesssim 1.4 /(10 \mathrm{TeV})^{2} .
$$

This interval corresponds to a prediction for $R(\pi)$ of

$$
1.0004 \lesssim R(\pi) \lesssim 1.0009
$$

at the $95 \%$ C.L. This prediction can be tested by the forthcoming results of PEN [42] and PiENu [43] experiments which anticipate in the near future an improvement by more than a factor of 3 . Furthermore, the proposed $\mathrm{PiENuXe}$ aims at an order of magnitude improvement in sensitivity compared to the current experimental result.

\section{CONCLUSIONS AND OUTLOOK}

While convincing hints for the violation of lepton flavor universality have been accumulating within recent years by low-energy precision experiments, corresponding signals in high-energy searches at the Large Hadron Collider have not been found in the past. However, recently, a nonresonant dilepton analysis of CMS showed an excess in electrons compared to muons. Even though CMS does not explicitly state that there is an excess, it is interesting that the surplus of events is the bins with the highest invariant mass of the lepton pair (as expected if it is due to a heavy new physics contribution) and both ATLAS and HERA also observe more electron events than expected in similar analyses.

Furthermore, the Cabibbo-angle anomaly (the deficit in first row and first column CKM unitarity at the $3 \sigma$ level) can be addressed via a tree-level new physics contribution to beta decays. Such an explanation must involve the operator $\left[Q_{\ell q}^{(3)}\right]_{1111}$ of the Standard Model effective field theory which leads to an effect in nonresonant dielectron searches at the LHC with a signal strength compatible with the one found by CMS. In fact, we find that a combined explanation of CMS data and the Cabibbo-angle anomaly with this operator can improve the total SM $\chi^{2}$ by more than 20 , corresponding to a pull of $\approx 4.5 \sigma$, while respecting the bounds from the corresponding ATLAS search. Note that

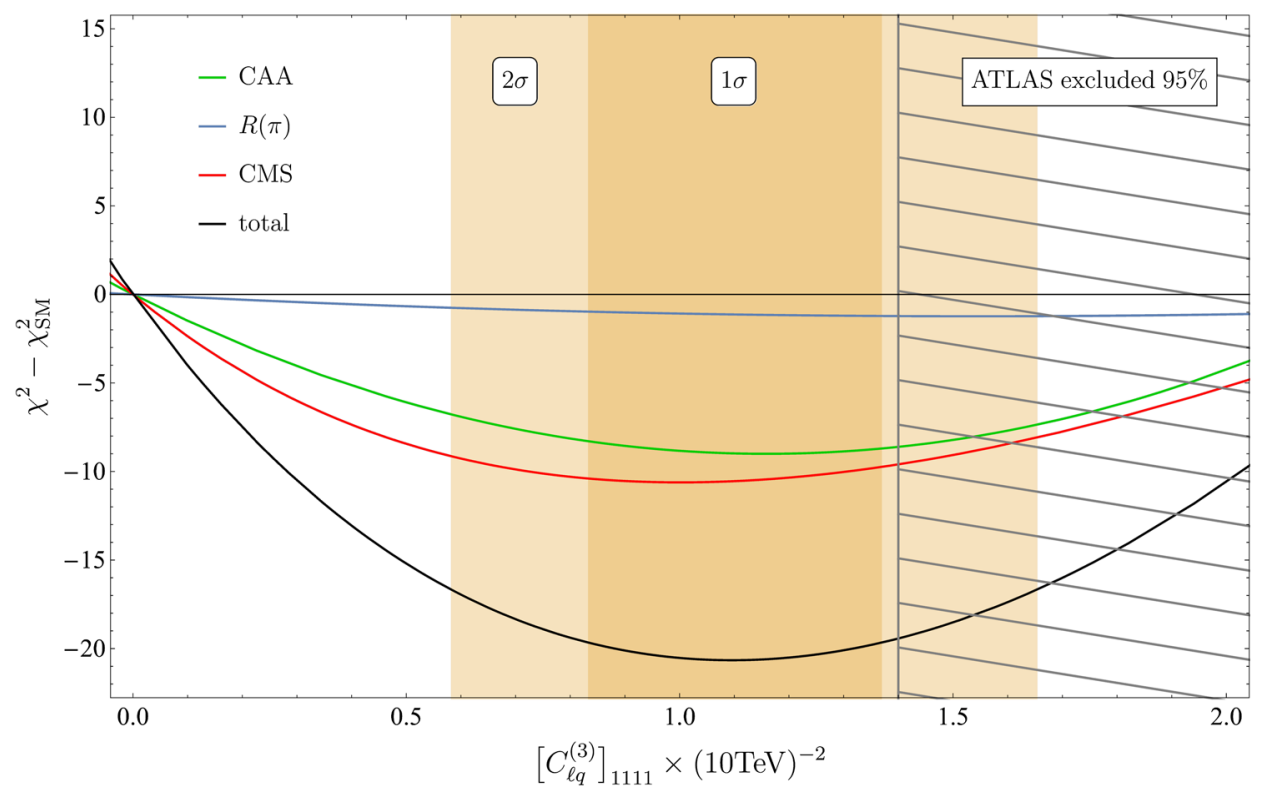

FIG. 2. $\Delta \chi^{2}=\chi^{2}-\chi_{\text {SM }}^{2}$ as a function of the Wilson coefficient $\left[C_{\ell q}^{(3)}\right]_{1111}$ for the fits including only $R(\pi)$ (blue), the CAA (green), the CMS analysis of dilepton pairs (red), and the combination of them (black) with the dark orange region showing the $1 \sigma$ and $2 \sigma$ regions of the combined fit. The hatched region is excluded at 95\% C.L. from the nonresonant dilepton search of ATLAS (not included in the $\chi^{2}$ function). The best fit point for the combined fit (black) is at $\left[C_{\ell q}^{(3)}\right]_{1111} \approx 1.1 /(10 \mathrm{TeV})^{2}$ where $\chi^{2}-\chi_{\mathrm{SM}}^{2} \approx-20$. 
this constitutes the first combined explanation of lepton flavor universality violation in low-energy precision observables and high-energy searches at the LHC.

Our scenario can be tested by analyses of the forthcoming LHC-Run 3 data and, to an even better degree, at the high luminosity LHC or the Large Hadron electron Collider [78]. Moreover improvements in the determinations of $V_{u s}, V_{u d}$, and also $V_{c d}$, both on the theoretical and experimental side (see Refs. [38,54] for a more detailed discussion), will scrutinize the deficit in first row and column CKM unitarity. In addition, we predict that in our setup $1.0004 \lesssim R(\pi) \lesssim 1.0009$ at $95 \%$ C.L. which can soon be tested by the PEN and PiENu experiments.
Furthermore, as we performed an effective field theory analysis, this opens up new possibilities in model building where e.g., $C_{\ell q}^{(3)}$ could be generated by a vector triplet, allowing for correlations with $b \rightarrow s \ell^{+} \ell^{-}$data [57].

\section{ACKNOWLEDGMENTS}

We thank Emanuele Bagnaschi, Noam Tal Hod, Massimiliano Procura, Oliver Fischer, and Daniel Lechner for useful discussions. This work is supported by a Professorship Grant (PP00P2_176884) of the Swiss National Science Foundation. A. C. thanks CERN for the support via the scientific associate program.
[1] R. Aaij et al. (LHCb Collaboration), J. High Energy Phys. 06 (2014) 133.

[2] R. Aaij et al. (LHCb Collaboration), Phys. Rev. Lett. 113, 151601 (2014).

[3] R. Aaij et al. (LHCb Collaboration), J. High Energy Phys. 09 (2015) 179.

[4] R. Aaij et al. (LHCb Collaboration), J. High Energy Phys. 02 (2016) 104.

[5] V. Khachatryan et al. (CMS Collaboration), Phys. Lett. B 753, 424 (2016).

[6] E. Auge, J. Dumarchez, and J.e.a. Tran Thanh Van (ATLAS Collaboration) (2017), https://inspirehep.net/ literature/1589906.

[7] The CMS Collaboration, Report No. CMS-PAS-BPH-15008, 2017.

[8] R. Aaij et al. (LHCb Collaboration), J. High Energy Phys. 08 (2017) 055.

[9] J. P. Lees et al. (BABAR Collaboration), Phys. Rev. Lett. 109, 101802 (2012).

[10] J. P. Lees et al. (BABAR Collaboration), Phys. Rev. D 88, 072012 (2013).

[11] R. Aaij et al. (LHCb Collaboration), Phys. Rev. Lett. 115, 111803 (2015); 115, 159901(E) (2015).

[12] R. Aaij et al. (LHCb Collaboration), Phys. Rev. D 97, 072013 (2018).

[13] R. Aaij et al. (LHCb Collaboration), Phys. Rev. Lett. 120, 171802 (2018).

[14] A. Abdesselam et al. (Belle Collaboration), arXiv: 1904.08794.

[15] G. W. Bennett et al. (Muon g-2 Collaboration), Phys. Rev. D 73, 072003 (2006).

[16] B. Capdevila, A. Crivellin, S. Descotes-Genon, J. Matias, and J. Virto, J. High Energy Phys. 01 (2018) 093.

[17] W. Altmannshofer, P. Stangl, and D. M. Straub, Phys. Rev. D 96, 055008 (2017).

[18] G. D’Amico, M. Nardecchia, P. Panci, F. Sannino, A. Strumia, R. Torre, and A. Urbano, J. High Energy Phys. 09 (2017) 010.
[19] M. Ciuchini, A. M. Coutinho, M. Fedele, E. Franco, A. Paul, L. Silvestrini, and M. Valli, Eur. Phys. J. C 77, 688 (2017).

[20] G. Hiller and I. Nisandzic, Phys. Rev. D 96, 035003 (2017).

[21] L.-S. Geng, B. Grinstein, S. Jäger, J. M. Camalich, X.-L. Ren, and R.-X. Shi, Phys. Rev. D 96, 093006 (2017).

[22] T. Hurth, F. Mahmoudi, D. M. Santos, and S. Neshatpour, Phys. Rev. D 96, 095034 (2017).

[23] A. K. Alok, B. Bhattacharya, A. Datta, D. Kumar, J. Kumar, and D. London, Phys. Rev. D 96, 095009 (2017).

[24] M. Algueró, B. Capdevila, A. Crivellin, S. Descotes-Genon, P. Masjuan, J. Matias, M. N. Brunet, and J. Virto, Eur. Phys. J. C 79, 714 (2019); 80, 511(A) (2020).

[25] J. Aebischer, W. Altmannshofer, D. Guadagnoli, M. Reboud, P. Stangl, and D. M. Straub, Eur. Phys. J. C 80, 252 (2020).

[26] M. Ciuchini, A. M. Coutinho, M. Fedele, E. Franco, A. Paul, L. Silvestrini, and M. Valli, Eur. Phys. J. C 79, 719 (2019).

[27] M. Ciuchini, M. Fedele, E. Franco, A. Paul, L. Silvestrini, and M. Valli, Phys. Rev. D 103, 015030 (2021).

[28] Y. Amhis et al. (HFLAV Collaboration), Eur. Phys. J. C 77, 895 (2017).

[29] C. Murgui, A. Peñuelas, M. Jung, and A. Pich, J. High Energy Phys. 09 (2019) 103.

[30] R.-X. Shi, L.-S. Geng, B. Grinstein, S. Jäger, and J. M. Camalich, J. High Energy Phys. 12 (2019) 065.

[31] M. Blanke, A. Crivellin, T. Kitahara, M. Moscati, U. Nierste, and I. Nišandžić, Phys. Rev. D 100, 035035(A) (2019).

[32] S. Kumbhakar, A. K. Alok, D. Kumar, and S. U. Sankar, Proc. Sci., EPS-HEP2019 (2020) 272.

[33] T. Aoyama et al., Phys. Rep. 887, 1 (2020).

[34] B. Belfatto, R. Beradze, and Z. Berezhiani, Eur. Phys. J. C 80, 149 (2020).

[35] Y. Grossman, E. Passemar, and S. Schacht, J. High Energy Phys. 07 (2020) 068.

[36] C.-Y. Seng, X. Feng, M. Gorchtein, and L.-C. Jin, Phys. Rev. D 101, 111301 (2020). 
[37] A. M. Coutinho, A. Crivellin, and C. A. Manzari, Phys. Rev. Lett. 125, 071802 (2020).

[38] A. Crivellin and M. Hoferichter, Phys. Rev. Lett. 125, 111801 (2020).

[39] A. M. Sirunyan et al. (CMS Collaboration), J. High Energy Phys. 07 (2021) 208.

[40] G. Aad et al. (ATLAS Collaboration), J. High Energy Phys. 11 (2020) 005.

[41] H. Abramowicz et al. (ZEUS Collaboration), Phys. Rev. D 99, 092006 (2019).

[42] C. J. Glaser et al. (PEN Collaboration), in Proceedings of the 13th Conference on the Intersections of Particle and Nuclear Physics (2018), https://inspirehep.net/literature/ 1706145.

[43] R. E. Mischke et al. (PiENu Collaboration), in Proceedings of the 13th Conference on the Intersections of Particle and Nuclear Physics (2018), https://inspirehep.net/literature/ 1695835.

[44] W. J. Marciano and A. Sirlin, Phys. Rev. Lett. 96, 032002 (2006).

[45] C.-Y. Seng, M. Gorchtein, H. H. Patel, and M. J. Ramsey-Musolf, Phys. Rev. Lett. 121, 241804 (2018).

[46] C. Y. Seng, M. Gorchtein, and M. J. Ramsey-Musolf, Phys. Rev. D 100, 013001 (2019).

[47] M. Gorchtein, Phys. Rev. Lett. 123, 042503 (2019).

[48] A. Czarnecki, W. J. Marciano, and A. Sirlin, Phys. Rev. D 100, 073008 (2019).

[49] L. Hayen, Phys. Rev. D 103, 113001 (2021).

[50] J. C. Hardy and I. S. Towner, Phys. Rev. C 102, 045501 (2020).

[51] M. Moulson, Proc. Sci., CKM2016 (2017) 033 [arXiv: 1704.04104].

[52] Y. S. Amhis et al. (HFLAV Collaboration), Eur. Phys. J. C 81, 226 (2021).

[53] P. A. Zyla et al. (Particle Data Group), Prog. Theor. Exp. Phys. 2020, 083C01 (2020).

[54] A. Crivellin, M. Hoferichter, and C. A. Manzari, Phys. Rev. Lett. 127, 071801 (2021).

[55] B. Belfatto and Z. Berezhiani, J. High Energy Phys. 10 (2021) 079.

[56] D. A. Bryman and R. Shrock, Phys. Rev. D 100, 073011 (2019).
[57] B. Capdevila, A. Crivellin, C. A. Manzari, and M. Montull, Phys. Rev. D 103, 015032 (2021).

[58] M. Endo and S. Mishima, J. High Energy Phys. 08 (2020) 004.

[59] A. Crivellin, F. Kirk, C. A. Manzari, and M. Montull, J. High Energy Phys. 12 (2020) 166.

[60] M. Kirk, Phys. Rev. D 103, 035004 (2021).

[61] A. K. Alok, A. Dighe, S. Gangal, and J. Kumar, Nucl. Phys. B971, 115538 (2021).

[62] A. Crivellin, C. A. Manzari, M. Alguero, and J. Matias, Phys. Rev. Lett. 127, 011801 (2021).

[63] A. Crivellin, F. Kirk, C. A. Manzari, and L. Panizzi, Phys. Rev. D 103, 073002 (2021).

[64] A. Crivellin, D. Müller, and L. Schnell, Phys. Rev. D 103, 115023 (2021).

[65] B. Grzadkowski, M. Iskrzynski, M. Misiak, and J. Rosiek, J. High Energy Phys. 10 (2010) 085.

[66] E. Eichten, I. Hinchliffe, K. D. Lane, and C. Quigg, Rev. Mod. Phys. 56, 579 (1984); 58, 1065(A) (1986).

[67] E. Eichten, K. D. Lane, and M. E. Peskin, Phys. Rev. Lett. 50, 811 (1983).

[68] M. Aaboud et al. (ATLAS Collaboration), J. High Energy Phys. 10 (2017) 182.

[69] J. M. Campbell, J. W. Huston, and W. J. Stirling, Rep. Prog. Phys. 70, 89 (2007).

[70] D. B. Clark, E. Godat, and F. I. Olness, Comput. Phys. Commun. 216, 126 (2017).

[71] A. Greljo and D. Marzocca, Eur. Phys. J. C 77, 548 (2017).

[72] G. Czapek et al., Phys. Rev. Lett. 70, 17 (1993).

[73] D. I. Britton et al., Phys. Rev. Lett. 68, 3000 (1992).

[74] D. A. Bryman, R. Dubois, T. Numao, B. Olaniyi, A. Olin, M. S. Dixit, D. Berghofer, J. M. Poutissou, J. A. Macdonald, and B. C. Robertson, Phys. Rev. Lett. 50, 7 (1983).

[75] V. Cirigliano and I. Rosell, Phys. Rev. Lett. 99, 231801 (2007).

[76] A. Aguilar-Arevalo et al. (PiENu Collaboration), Phys. Rev. Lett. 115, 071801 (2015).

[77] M. Tanabashi et al. (Particle Data Group), Phys. Rev. D 98, 030001 (2018).

[78] P. Agostini et al. (LHeC Collaboration, FCC-he Study Group), arXiv:2007.14491. 